\title{
Gravitational Wave Generation and Detection Using Acoustic Resonators and Coupled Resonance Chambers
}

\author{
R. Clive Woods ${ }^{1}$ and Robert M.L. Baker, Jr. ${ }^{2}$ \\ ${ }^{1}$ Department of Electrical and Computer Engineering and Microelectronics Research Center, \\ 2128 Coover Hall, Iowa State University, Ames, Iowa 50011-3060 \\ ${ }^{2}$ GRAVWAVE ${ }^{\circledR}$ LLC, Playa del Rey, California, 90293-7856 \\ 1(515) 294 3310,Fax(515) 294 8432,cwoods@iastate.edu
}

\begin{abstract}
An experiment is described for the generation and detection of High-Frequency Gravitational Waves (HFGWs) in the laboratory utilizing acoustic piezoelectric resonators for generation, and coupled resonance chambers for detection. Film Bulk Acoustic Resonators or FBARs (similar to those utilized in commercial cellular telephones) energized by magnetrons (similar to those utilized in microwave ovens) are distributed in a ring-shaped array several hundred meters in diameter. The magnetrons are phase locked and are sufficient in number to energize millions of FBARs fabricated on thousands of wafers. The FBARs produce jerks (time rate of change of acceleration or a third time derivative motion imparted to their electrode masses) at a frequency of $2.45 \mathrm{GHz}$. The resulting $4.9 \mathrm{GHz} \mathrm{HFGW}$ is focused at the center of a segmented or asymmetrical ring of FBARs and is concentrated there by a high-temperature superconductor (HTSC) to generate a HFGW flux on the order of $17 \mathrm{~mW} \mathrm{~m}^{-2}$ to $7 \mathrm{~W} \mathrm{~m}$. A miniature version of an existing HFGW detector designed at INFN Genoa, Italy, consisting of a pair of coupled HTSC-surfaced resonance chambers (about one centimeter in diameter) will be situated in the middle, having their axes perpendicular to the plane of the ring of FBARs. Alternatively, a resonance-loop chamber detector, similar to that developed at Birmingham University, U.K., could be utilized. Details of the experiment and challenges to be met in its design as well as applications to space technology are discussed.
\end{abstract}

\section{INTRODUCTION}

In order to understand gravitational waves, consider first water waves. These waves are disturbances or undulations on or in a medium: the water. They exhibit an amplitude or height and a frequency that is dependent on the time between the passages of wave crests past some fixed point. They can be sensed, for example, by the motion of a buoy or leaf on the water's surface. Sound waves propagate as compressions and rarefactions in a gas medium usually air. They have amplitude according to the pressure difference of the compressions and rarefactions and a frequency that is related to the time between adjacent wave pressure crests. They can be sensed by, for example, the motion of a diaphragm in a microphone. Electromagnetic (EM) waves like radio, microwaves, light, or X-rays depend upon the propagation of variations in an EM field that also exhibit a frequency like other waves, but they propagate at a constant speed, $c \approx 3 \times 10^{8} \mathrm{~m} \mathrm{~s}^{-1}$ or the "speed of light." Also there is no true medium for EM waves. Such EM waves can be sensed by, for example, the motion of electrons in a photocell. Einstein (1915) theorized a revolutionary space-time fabric or continuum in his general theory of relativity. He called the undulations or waves propagating in this fabric "gravitational waves" (Einstein, 1916). He theorized that they propagate at the speed of light and could be generated, for example, by orbiting stars, spinning rods, or dumbbells. Gravitational waves (GWs) can be sensed by, for example, the change in lengths measured by extremely sensitive interferometers, piezoelectric crystals, superconductors, or resonance chambers. They have never been directly sensed, however, and some scientists believed that these waves were unobservable artefacts of Einstein's theory. The indirect evidence obtained by Taylor (1994) and R.A. Hulse concerning their observations of a contracting binary star pair or pulsar PSR 1913+16, which perfectly matched Einstein's GW theory, garnered them the 1993 Nobel Prize and skepticism concerning GWs evaporated. According to a set of definitions provided by Hawking and Israel (1979), HighFrequency Gravitational Waves (HFGWs) have frequencies in excess of $100 \mathrm{kHz}$ and have the most promise for terrestrial generation and practical, scientific and commercial applications. Low-Frequency Gravitational Waves (LFGWs) typically generated by most astrophysical sources are expected to be detected by interferometric and 
resonance devices whose technology is totally different from the technology of high-frequency detector devices - as different as AC-motor technology is from microwave-transmitter technology. Thus LFGW detectors such as LIGO, VIRGO, LISA, DECIGO (Japan), and CEGO (China) are useless for HFGW detection.

Since 2001, studies of HFGWs have intensified, including generation, detection, and engineering application. In 2003 the first meeting of the International High-Frequency Gravitational Waves Working Group, sponsored by MITRE Corporation, McLean (VA), was held (Baker, 2003a). This conference attracted 25 scientific papers from nine countries, and discussed many HFGW concepts. It also displayed the strong international activity in this area.

\section{SIGNIFICANCE OF HFGW GENERATION}

There has never been any significant effort to generate and detect gravitational waves in the laboratory since only the theory has been considered so far. The experiment proposed here will lead to revolutionary ways in which space propulsion and communication are accomplished 10 to 40 years from now. Remote HFGW generators will modify the gravitational field near an object or spacecraft: "Since it has definite energy, the gravitational wave is itself the source of some additional gravitational field...its field is a second-order effect...But in the case of high-frequency gravitational waves the effect is significantly strengthened..." (Landau and Lifshitz, 1975, p. 349). Thus it is possible to change the gravitational field near an object by means of HFGWs and move or perturb it. And, thus there exists a completely new means to propel a spacecraft. The significance of such propellantless propulsion has been considered by many authors. HFGW generators may also be used as a completely new means of space communication. T.A. Prince (2002) recently commented: "Of the applications (of HFGWs), communication would seem to be the most important. Gravitational waves have a very low cross section for absorption by normal matter and therefore high-frequency waves could, in principle, carry significant information content with effectively no absorption unlike any electromagnetic waves." Such a HFGW communication system would represent the ultimate wireless system - point-to-multipoint $\mathrm{PHz}$ communication without the need for expensive enabling infrastructure, that is, no need for fiber-optic cable, satellite transponders, or microwave relays.

\section{PROPOSED GENERATOR CONCEPT}

The concept proposed here is to generate and detect HFGWs in the laboratory using a novel arrangement of Film Bulk Acoustic Resonators (FBARs). They will be energized by phase locked magnetrons similar to those utilized in ordinary microwave ovens. The magnetrons must all be phase-locked; it is proposed to achieve this using a similar approach to that in phased array antenna designs (Gordon, 2004). The details of the derivation of the HFGW generation equations, summarized below, are given by Baker $(2000,2002)$, and the concept of generating HFGWs by an array of micro-devices is described by Baker (2003b, 2004). From Einstein's General Theory of Relativity the power of a $\mathrm{GW}$ generator is given by his quadrupole equation. This equation can be written as

$$
P(r, \Delta f, \Delta t)=1.76 \times 10^{-52}(2 r \Delta f / \Delta t)^{2} \mathrm{~W},
$$

where $r$ is the radius of gyration of a mass (or system of masses) in meters (about $300 \mathrm{~m}$ to $3 \mathrm{~km}$ for the experiment proposed here), $\Delta f$ is an incremental increase in force in newtons acting on a mass during an incremental time period $\Delta t$ in seconds, commonly referred to as a "jerk". This is the jerk formulation of the quadrupole equation. For a continuous train of jerks the frequency is $v=1 / \Delta t$, and Eq. (1) can be phrased as a function of HFGW frequency as

$$
P(r, \Delta f, v)=1.76 \times 10^{-52}(2 r v \Delta f)^{2} \mathrm{~W} .
$$

An impractical method of generating HFGWs is to rotate a rod so that it radiates energy through this quadrupole HFGW generation mechanism. This is impractical because it has long been known that rotation of any material at sufficiently high speed for efficient HFGW generation according to Eq. (2) is impossible; at much lower rotation speeds than are needed for efficient HFGW generation, centrifugal force causes the rotating mass to disintegrate. This is the origin of the widely-held view that generation of GWs is impossible outside of astronomical bodies. The basic concept proposed here is to utilize piezoelectric mechanical resonators, specifically Film Bulk Acoustic Resonators (FBARs) similar to those utilized in cellular telephones, energized by magnetrons similar to those utilized in microwave ovens, to produce high frequency jerks in the vibrational elements of the FBARs and thereby 
generate HFGWs. An FBAR (Fig. 1) is a mechanical (acoustic) resonator consisting of a vibrating membrane (typically around $100 \times 100 \mu \mathrm{m}^{2}$ in planform, and around $1 \mu \mathrm{m}$ thickness), fabricated using well-established integrated-circuit microfabrication technology. The vibrating membrane is actuated piezoelectrically, typically using aluminum nitride (AIN) as the piezoelectric excitation material. These devices have recently been highly developed as cost-effective RF filters mainly for use in commercial cellular telephones, in which high- $Q$ resonators are needed for the typical carrier frequency band around $1.9 \mathrm{GHz}$. The vibrating membrane in an FBAR will also form an ideal vibrating mass for the present application, at slightly higher frequency. Exploratory FBAR devices have been fabricated operating at frequencies up to 7.5GHz (Ruby and Merchant, 1994; Lakin et al., 2001).

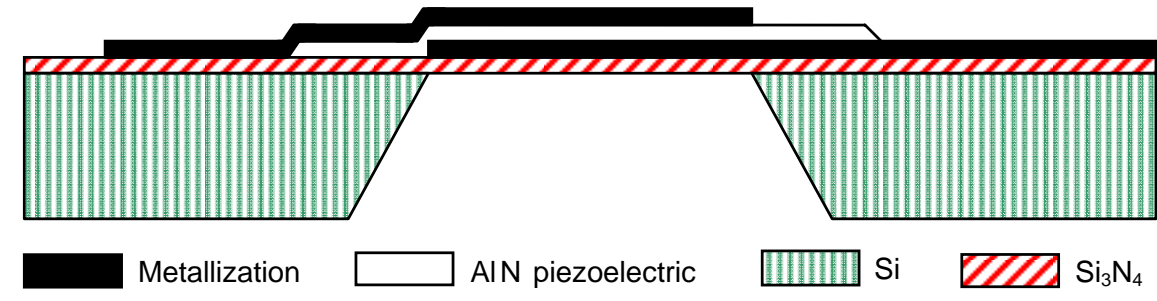

FIGURE 1. Basic FBAR Construction (Side View, Not to Scale).

The FBARS will be arranged in a circle and each vibrating element must vibrate in phase with all the rest. In this way, a segmented or asymmetrical ring of masses all oscillating at the same frequency will be produced which reproduces the essentials of an asymmetrical toroidal mass oscillating or rotating at the same frequency, except that no centrifugal forces are generated so that the system will not be torn apart by centrifugal forces. The FBAR jerks tangential to the stationary rim are exactly analogous to jerks occurring in a solid asymmetrical toroid. The essential concept here is that a collection of asymmetrical rotating masses, with their attendant centrifugal-force jerks, is emulated by a series of fixed masses that are jerked by piezoelectric forces - energizable elements (FBARs) acted upon by energizing elements (magnetrons). Three important points should now be made:

- The energizable element's action/reaction does not cancel out during GW generation (Einstein and Rosen, 1937), but the energizable elements should be asymmetrically distributed.

- The acceleration can be well over $100 \mathrm{~g}$ and the quadrupole equation will still be valid: e.g., the acceleration of the contracting neutron star pair or pulsar PSR 1913+16 is $112 g$ at periastron (Baker, 2000, 2002).

- $\Delta f$ need $n o t$ be gravitational force (Einstein, 1916; Infeld, quoted by Weber (1964, p. 97)). EM forces are $\sim 10^{35}$ times larger than typical gravitational forces and so will give significant advantage in laboratory GW generation. As Weber (1964) points out: "The nongravitational forces play a decisive role in methods for detection and generation of gravitational waves...".

The generated HFGWs must then be focused to the smallest area possible at the focal point at the center of the segmented or asymmetrical ring of FBARs in order to achieve a large HFGW flux (energy/area) for detection. Commercial microwave oven magnetrons are proposed for this experiment because of their low cost in bulk quantities. These typically operate at $2.45 \mathrm{GHz}$. Similarly, suitable FBARs may be fabricated cheaply in large quantities and a small design change will produce resonance at $2.45 \mathrm{GHz}$ instead of the cellular telephone band at $1.9 \mathrm{GHz}$. Therefore, the operating frequency of the system will be $2.45 \mathrm{GHz}$, giving a gravitational frequency $v=$ 4.9GHz and a gravitational wavelength of $\lambda=c / v \approx 6.1 \mathrm{~cm}$. The FBARs need to be accurately oriented around the ring, within a fraction of one HFGW wavelength, and their lines of action (i.e., of the jerks of their vibrational elements) must be coplanar and accurately tangential to the stationary ring to a small fraction of a HFGW wavelength. This means that positional accuracy within at least $1 \mathrm{~cm}$ must be rigorously maintained. Sketches of the emulation of rotating masses $(\delta m)$ by a circular array of FBARs are shown in Fig. 2. Fig. 2(a) depicts two masses from an asymmetrical toroid rotating uniformly, and Fig. 2(b) depicts a segmented or asymmetrical ring of FBARs (each located on a circle of radius $r$ ) that produce the same HFGW effect.

\section{ANALYSIS OF THE FLUX OF A HFGW GENERATOR}

From Landau and Lifshitz (1975, p. 356) it can be shown that the radiation pattern or specific angular intensity, $I$, for a sequentially energized ring of asymmetrically distributed energizable elements (e.g., FBARs) can be expressed 


$$
I=P_{\mathrm{o}} \times 7.55 \times 10^{-6}\left(1+6 \cos ^{2} \theta+\cos ^{4} \theta\right) \mathrm{W} \mathrm{deg}{ }^{-2},
$$

where $P_{\mathrm{o}}$ is the power of the $\mathrm{GW}$ generator in $\mathrm{W}$, and $\theta$ is the polar angle measured from the central axis (perpendicular to the plane of motion through the ring's center). In three dimensions the radiation pattern looks like two candelabra bulbs joined at their bases, or a dumbbell. This is shown in Fig. 2(b). Coincidentally the detector geometry resembles a constant potential surface in this radiation pattern and this provides insights in designing new and different detectors tailored to specific radiation patterns. The GW flux has been computed at a "cap" on each side of the focus defined by a cone having an altitude $A$ and a $\pm 10^{\circ}$ vertex angle intersecting the three-dimensional radiation pattern. The GW flux, $F_{ \pm 10^{\circ}}$, in either of the caps is given by

$$
F_{ \pm 10^{\circ}}=P_{\mathrm{o}} \times 2.54(0.282 / A)^{2} \mathrm{~W} \mathrm{~m}^{-2}
$$

(where $A=0.282 \mathrm{~m}$ yields a $1 \mathrm{~m}^{2}$ area sphere). The closer the detector is to the focus along the central axis the better.

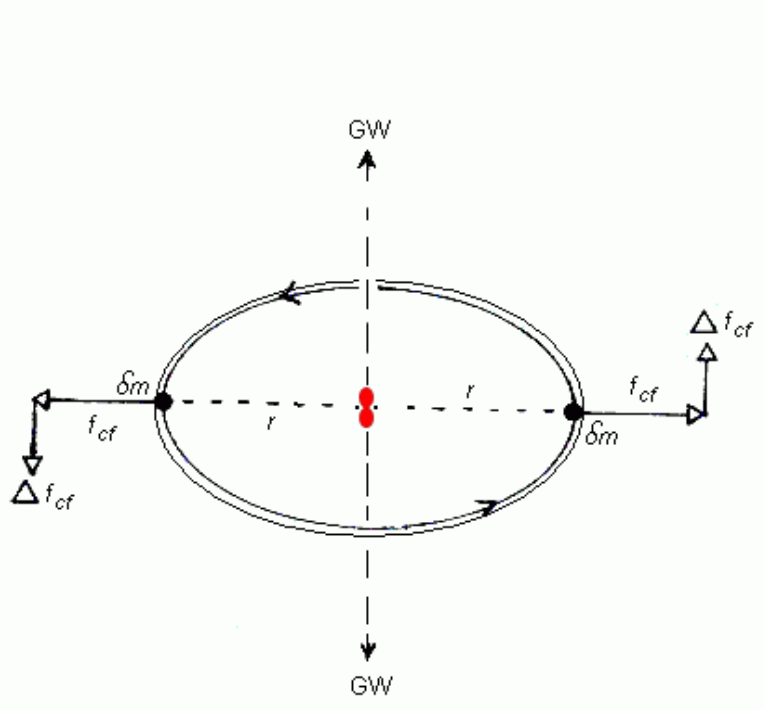

(a)

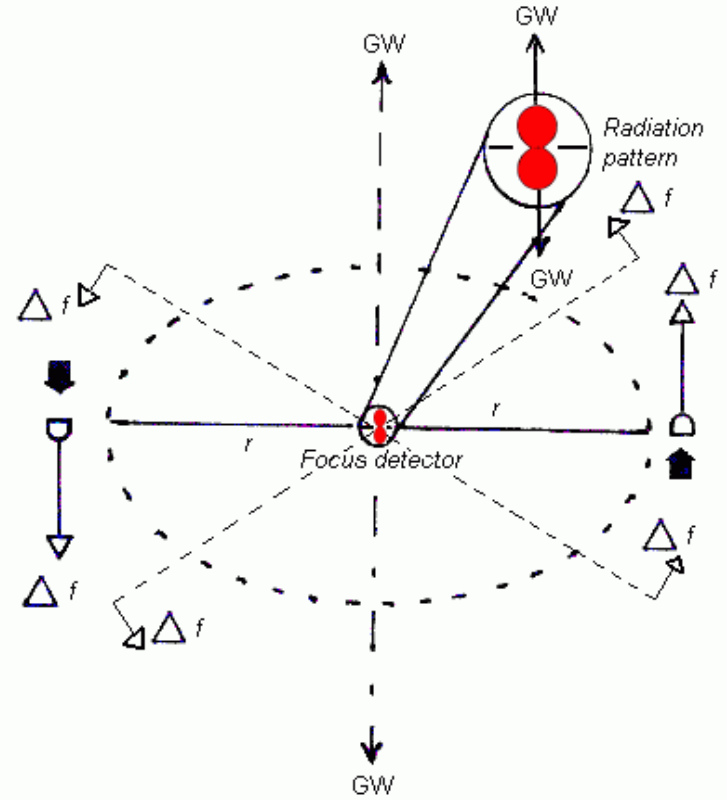

(b)

FIGURE 2. Emulation of a Rotating Asymmetrical Set of Masses by a Fixed Asymmetrical Circular Array of FBARs.

The HFGW power from a HFGW generator is proportional to $v^{2}$ according to Einstein's quadrupole relation, Eq. (2). Li and Torr (1992) showed that a superconductor (SC) has strong GW refractive properties that may be used to make a lens for GWs (although this result was disputed by Kowitt (1994)). For a Gaussian beam, perfectly focused (convergence angle $90^{\circ}$ ), the diffraction-limited spot diameter is $2 \lambda / \pi$ (Saleh and Teich, 1991), and so the smallest area into which the GW power may be focused is $\lambda^{2} / \pi$ on both sides of the center; the total area is therefore $2 \lambda^{2} / \pi$. (The Gaussian diffraction formula is more nearly applicable to the present case than is the plane-wave formula.) Since $\lambda^{2}=c^{2} / v^{2}$, the diffraction-limited focus area is inversely proportional to $v^{2}$, so the HFGW flux (power/area) at the focus is proportional to $v^{4}$. Away from the focus the flux will be significantly reduced, so the challenge is to site the HFGW detector as close as possible to the focus and to make the active detection surface or volume coincident with the diffraction-limited focus. [There may also be Fresnel reflection at the air-superconductor surfaces that needs to be addressed theoretically (Baker et al., 2005). This could be both detrimental if it causes excessive HFGW power loss at each lens surface, or advantageous if it enables reflection and refocusing of HFGW energy otherwise lost because it is travelling away from the detector.] Consider an aerospace communications HFGW generation/detection system in which the generated HFGWs are formed into a collimated beam by a HTSC lens and that at some distance away there is another HTSC lens to concentrate the HFGWs at the detector. The two lenses are diffraction-limited. The beam area of the HFGW beam due to diffraction is inversely proportional to $v^{2}$. The second lens concentrates the intercepted HFGWs at the detector into a diffraction-limited area, also inversely proportional to $v^{2}$. Therefore, the efficiency of this transmitter/receiver system is proportional to $v^{6}$ (Baker, 2000). If the detector is located at the focus of the HFGW generator, then the HTSC surrounding the focus concentrates the HFGWs there by a factor equal to the refractive index $=400 \pm 200$ ( $\mathrm{Li}$ and Torr, 1992, p. 5491; Li, 2002) in each axis. The GW 
wavelength is $(400 \pm 200) \times$ smaller in the HTSC and consequently the diffraction-limited spot size will be $400^{2} \times$ smaller than in free space. In this case the HFGW signal at the detector is proportional to $v^{4}$. [In fact, the efficiency is slightly reduced because the sensitivity of many GW detectors is proportional either to $1 / \sqrt{ } v$ or to $1 / v$, so the overall efficiency will be proportional to $v^{5.5}, v^{5}, v^{3.5}$, or $v^{3}$.] Therefore, there is an enormous advantage in working at the highest frequency possible.

\section{ESTIMATE OF PERFORMANCE OF FBARS AS HFGW GENERATORS}

In this section a simple model of a HFGW generator assembled from FBARs is presented. Piezoelectric excitation of a small acoustic (mechanical) resonator is far superior to EM excitation at frequencies above tens of $\mathrm{kHz}$ because there are no inductive losses in a typical piezoelectric device. However, the acoustic electrodes must be small so as not to introduce excessive capacitive loss. Existing high-performance FBARs (Ruby and Merchant, 1994; Lakin et al., 2001) demonstrate the feasibility of low-loss operation of this class of device around $2 \mathrm{GHz}$.

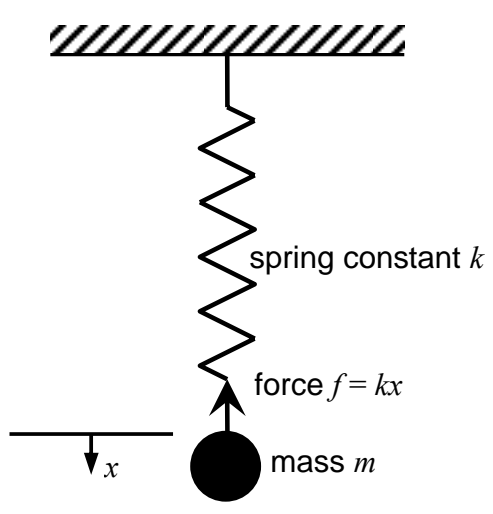

FIGURE 3. Simple Model of Acoustic Resonator.

The basic construction of an FBAR is illustrated in Fig. 1. Accurate modelling of a membrane resonator is a difficult task but here a very simple analog is used to estimate the basic performance of this device as a generator of HFGW. First, the stored energy per FBAR is estimated. The resonance quality factor is given by the well-known result $Q=$ $\omega_{\mathrm{o}} \times($ stored energy $) /($ power applied $)$, where $\omega_{\mathrm{o}}$ is the resonant angular frequency, and so the stored energy is

$$
W=Q \times(\text { power applied }) / \omega_{0} \text {. }
$$

To calculate the force applied to one FBAR membrane, the simple model of an acoustic resonator shown in Fig. 3 is used here. The vibrational mode of an FBAR membrane is actually a low-order Lamb wave (Auld, 1990) although a simple approximation will be accurate enough for a first estimate. Mass $m$ fixed to the end of a spring of spring (force) constant $k$ oscillates at a natural angular frequency $\omega_{0}=(k / m)^{1 / 2}=2 \pi v_{0}$ so that

$$
k=\omega_{0}^{2} m \text {. }
$$

Second, the energy stored in the oscillation is given by $W=1 / 2 k x^{2}=1 / 2\left(k x_{0}\right)^{2} / k=1 / 2 f_{0}^{2} / k$ for an oscillation amplitude $x_{0}$ and maximum force $f_{\mathrm{o}}$, so that from Eq. (6) the maximum force applied to the mass is

$$
f_{\mathrm{o}}=(2 W k)^{1 / 2}=\left(2 W \omega_{\mathrm{o}}^{2} m\right)^{1 / 2}
$$

Suppose the total excitation power available is $P_{\text {in }}$ divided equally between $N$ FBARs. Then the power supplied to each FBAR is $P_{i n} / N$ (assuming, of course, no loss from power distribution or phase adjusting), so that from Eq. (5) the stored energy per FBAR is $W=Q P_{i n} /\left(N \omega_{0}\right)$. Combining this with Eq. (7) gives the force in each FBAR

$$
f_{\mathrm{o}}=\left(2 Q P_{\text {in }} \omega_{\mathrm{o}} m / N\right)^{1 / 2} \text {. }
$$

Therefore, the total force experienced by $N$ FBARs excited in phase is $\left(2 Q P_{i n} \omega_{0} m N\right)^{1 / 2}$. Note that the total force is proportional to $N^{1 / 2}$ rather than to $N$, because with more FBARs but fixed total input power $P_{\text {in }}$ then the power per FBAR is reduced. To maximize the total force, the largest possible number of FBARs is needed, operating at the highest possible frequency and largest possible input power. A typical FBAR has a resonance curve with a passband 
resonance width of $2 \Delta v=24 \mathrm{MHz}$ at a typical passband center frequency $v_{\mathrm{o}}=2 \mathrm{GHz}$ (Lakin et al., 2001). This gives $Q \approx 2000 / 24 \approx 100$. Mass $m$ is found from density $\times$ volume. The values density $=3000 \mathrm{~kg} \mathrm{~m}^{-3}$ (typical of materials comprising an FBAR membrane) and volume $=100 \times 100 \times 1 \mu \mathrm{m}^{3}$ (typical of an FBAR membrane resonant at $v_{\mathrm{o}}=$ $2 \mathrm{GHz}$ ) were estimated here, so that $m=30 \mathrm{ng}$. A typical FBAR takes up rather more area than its nominal membrane area $100 \times 100 \mu \mathrm{m}^{2}$ in a fabricated silicon wafer. Current silicon fabrication foundries can process 4" diameter (or larger) silicon wafers as standard. From these figures it is straightforward to calculate that, at a very conservative estimate, 6000 (or more) FBARs may easily be fabricated on one wafer (Lakin et al., 2001). The total force is proportional to $N^{1 / 2}$ and so the generated HFGW flux (from Eq. (2)) is proportional to $\left(N^{1 / 2}\right)^{2}=N$.

Suitable magnetrons giving $1 \mathrm{~kW}$ at $2.45 \mathrm{GHz}$ are readily available in bulk off-the-shelf at about US\$30 each, intended for OEM microwave oven use. FBARs are quite simple to fabricate using standard microelectronics procedures. For this application, custom-designed devices would be needed to match the magnetron frequency; a very rough estimate of their cost is US\$10 per wafer (in bulk quantities). (In fact it would be economically advantageous to use 6" or 8 " diameter wafers but here 4" wafers are assumed.) To find the best distribution of magnetrons and FBAR wafers, note that the HFGW flux is proportional to the product $\left(P_{i n} N\right)$; this product must be maximized subject to the constraint of minimum cost. This is an optimization problem and the solution is: equal sums should be spent on the magnetrons and on the FBAR wafers. Therefore, the optimum arrangement is to have each $1 \mathrm{~kW}$ magnetron drive three 4" FBAR wafers, assuming as an illustrative example the rough estimates of costs given above. This excitation corresponds to $\sim 56 \mathrm{~mW}$ per FBAR, well within the power-handling capacity of this type of device (typically $\sim 2 \mathrm{~W}$ per FBAR is reported by Ruby et al. (1999)).

Suppose that US\$600k, an arbitrarily chosen sum chosen purely as an example, is available for the total hardware cost of the magnetrons and FBAR wafers. The optimum design at this price consists of 10,000 magnetrons, costing US\$300k, driving a total of 30,000 FBAR wafers, also costing US\$300k. The magnetrons must be spaced nonuniformly around a large circle on level ground; its radius is arbitrarily chosen here to be $300 \mathrm{~m}$, so that on average the magnetrons are spaced apart by $\sim 19 \mathrm{~cm}$. Laser surveying devices would be necessary to align all the generating elements accurately towards the central focus within about $\pm 1 \mathrm{~cm}$. Thus there would be $\sim 1.8 \times 10^{8}$ FBARs. From Eq. (8), using $Q=100, P_{i n}=10 \mathrm{MW}$, and $\omega_{0}=2 \pi \times 2.45 \mathrm{GHz}$, the total force each cycle is $4 \times 10^{8} \mathrm{~N}$. The magnetron frequency, $2.45 \mathrm{GHz}$, corresponds to a generated HFGW frequency $4.9 \mathrm{GHz}$. From Eq. (2), the generated HFGW power is $P=250 \mathrm{pW}$. The wavelength of a $4.9 \mathrm{GHz} \mathrm{HFGW}$ is $\lambda=c / v \approx 6.1 \mathrm{~cm}$, and dividing by the area of the diffraction-limited spot size, $2 \lambda^{2} / \pi$, gives a HFGW flux of $0.1 \mu \mathrm{W} \mathrm{m} \mathrm{m}^{-2}$ in free space over a detector aperture placed at the center of the circular generator (above or below the focus point). Using a HTSC lens at the detection aperture to reduce the HFGW wavelength $400 \times\left(\mathrm{Li}\right.$ and Torr, 1992), making the diffraction-limited spot area $(400)^{2} \times$ smaller, gives a flux of $17 \mathrm{~mW} \mathrm{~m}^{-2}$.

\section{INFN GENOA DETECTOR}

This detector has been developed by Chincarini and Gemme (2003) of INFN, Genoa, Italy, and is based upon coupling superconducting radio frequency (RF) cavities and exploits the parametric energy conversion between two EM resonant modes. The interaction of the gravitational wave with the superconducting cavity walls induces a motion that is sensed by the EM field stored within the cavity. The energy conversion is maximized when the frequency of the wave is equal to the frequency difference of the cavity resonant modes. Several such devices have been produced by Chincarini, Gemme, and others, proving that this approach is feasible for HFGW detection in the $\mathrm{GHz}$ range. Detection at $4.9 \mathrm{GHz}$ (the frequency expected in the present proposed configuration) requires a cavity resonance in excess of this frequency, implying overall detector dimensions on the order of $\sim 2 \mathrm{~cm}$. In order to build a realistic detector, a suitable cavity shape has to be chosen. From quite general arguments a detector based on two coupled spherical cavities looks very promising (Fig. 4(a)). To match the frequency range relevant here, the mode splitting (i.e., the detection frequency) should cover the range $10 \mathrm{kHz}<\left(\omega_{2}-\omega_{1}\right) /(2 \pi)<3 \mathrm{GHz}$. The internal radius of the spherical cavities would be in the range $20-100 \mathrm{~mm}$, corresponding to a $\mathrm{TE}_{011}$ mode at frequency $\omega /(2 \pi) \approx 2$ $10 \mathrm{GHz}$. A tuning cell, or a superconducting bellow, will be inserted in the coupling tube between the two cavities, allowing variation of the coupling strength (i.e., the detection frequency) in a narrow range around the design value. The spherical resonators can be easily deformed in order to favor the field polarization suitable for GW detection: the optimal field spatial distribution is with the field axis of the two cavities orthogonal to each other (Fig. 4(b)). 


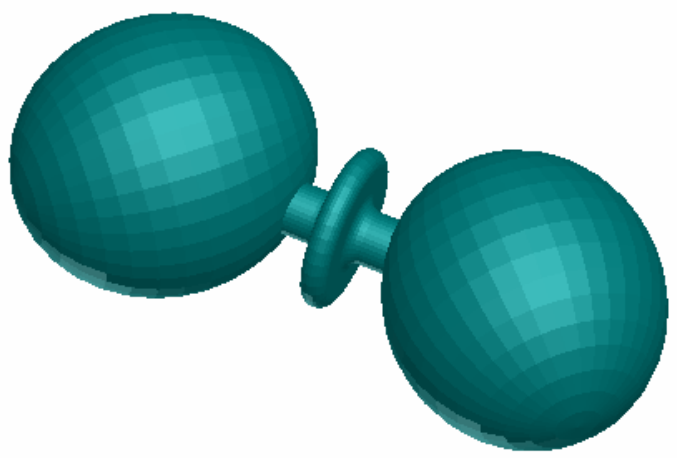

(a) Artist's Impression of the Coupled Spherical Cavities with Central Tuning Cell.

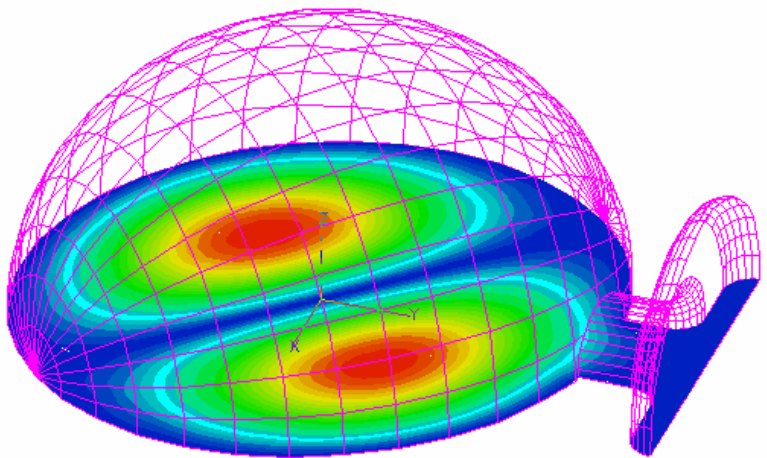

(b) Electric Field Magnitude of the $\mathrm{TE}_{011}$ Mode. Note the Alignment of the Field Axis.

FIGURE 4. INFN Genoa Detector (Chincarini and Gemme, 2003). Courtesy Dr. A. Chincarini.

\section{BIRMINGHAM UNIVERSITY DETECTOR}

The detector designed at Birmingham University, England, uses a resonant microwave loop-shaped chamber in which the interaction between HFGWs and the polarization vector of an EM wave is such that the polarization vector rotates about the direction of EM propagation (Cruise, 2000). When a resonance condition is established, the EM wave always experiences the same phase as the HFGW. The effect is cumulative and can be enhanced linearly by repeated EM circuits of a closed loop or waveguide. This detector essentially measures the curvature of spacetime and a prototype detector was fabricated by R. Ingley at Birmingham. The loop is about $1 \mathrm{~m}$ in length and can detect $200 \mathrm{MHz}$ HFGWs. Although the prototype dimensions are roughly $1 \mathrm{~m}$, the dimensions could be easily reduced in size to about $6 \mathrm{~cm}$ for $\mathrm{HFGW}$ detection at $4.9 \mathrm{GHz}$. The smaller the loop dimensions, the higher the gravitational-wave frequency that can be detected.

\section{DETECTING GENERATED HFGWS USING AVAILABLE DETECTORS}

Using flux compression by a HTSC lens, the HFGW flux produced by the generating arrangement described above is much greater than the detection limits of both the INFN Genoa and the Birmingham University detectors and should be easily detectable. These detectors, however, may not be quite sensitive enough to detect the corresponding flux generated in free space. At very little extra expense, the circle radius could be increased to $3 \mathrm{~km}$, giving a HFGW flux $10 \mu \mathrm{W} \mathrm{m}{ }^{-2}$ in free space or $1.7 \mathrm{~W} \mathrm{~m}^{-2}$ using a HTSC focusing lens. With doubled funding the number of magnetrons and FBARs might both be doubled to give $40 \mu \mathrm{W} \mathrm{m} ~^{-2}$ in free space or $7 \mathrm{~W} \mathrm{~m}^{-2}$ using a HTSC lens. These latter figures would be well within detection capabilities.

\section{CONCLUSIONS}

The basic generator proposed here consists of 10,000 phase-locked magnetrons, asymmetrically arranged in a circle of radius $300 \mathrm{~m}$, each producing $1 \mathrm{~kW}$ at $2.45 \mathrm{GHz}$; each supplies three FBAR wafers, each of 6,000 phase-coherent resonant FBARs. This produces a HFGW radiated power $250 \mathrm{pW}$ at $4.9 \mathrm{GHz}$. Focused to a diffraction-limited spot in free space this corresponds to a HFGW flux of $0.1 \mu \mathrm{W} \mathrm{m}{ }^{-2}$. This is not quite within the detection capabilities of current HFGW detectors but increases to well within the sensitivity range if HTSC is used to reduce the HFGW wavelength and hence reduce the area of the diffraction-limited spot, and $/$ or if the circle radius is increased to $3 \mathrm{~km}$.

\section{NOMENCLATURE}

$A=$ cone altitude (m)

$$
c=\text { speed of light }\left(\mathrm{m} \mathrm{s}^{-1}\right)
$$




$$
\begin{aligned}
f & =\text { force }(\mathrm{N}) \\
f_{\mathrm{o}} & =\operatorname{maximum} \text { force }(\mathrm{N}) \\
F & =\mathrm{GW} \text { flux }\left(\mathrm{W} \mathrm{m}^{-2}\right) \\
k & =\text { spring constant }\left(\mathrm{N} \mathrm{m}^{-1}\right) \\
Q & =\text { resonance quality factor } \\
m & =\text { mass }(\mathrm{kg}) \\
N & =\text { number of FBARs } \\
P & =\text { power }(\mathrm{W}) \\
P_{\text {in }} & =\text { total power available }(\mathrm{W})
\end{aligned}
$$

$$
\begin{aligned}
r & =\text { radius of gyration }(\mathrm{m}) \\
\Delta t & =\text { time increment }(\mathrm{s}) \\
W & =\text { stored energy }(\mathrm{J}) \\
x & =\text { spring extension }(\mathrm{m}) \\
\lambda & =\text { wavelength }(\mathrm{m}) \\
\Delta v & =\text { FBAR half-resonance width }(\mathrm{Hz}) \\
v & =\text { frequency }(\mathrm{Hz}) \\
v_{\mathrm{o}} & =\text { resonant center frequency }(\mathrm{Hz}) \\
\omega_{\mathrm{o}} & =\text { resonant angular frequency }\left(\mathrm{rad} \mathrm{s}^{-1}\right)
\end{aligned}
$$

\section{REFERENCES}

Auld B.A., Acoustic Fields and Waves in Solids, Vol. II 2nd. Ed., Krieger, Malabar, FL, 1990, p. 85.

Baker, R.M.L., Jr., "Preliminary Tests of Fundamental Concepts Associated with Gravitational-Wave Spacecraft Propulsion," in Proc. American Institute of Aeronautics and Astronautics, 2000-5250, Reston, VA, 2000.

Baker, R.M.L., Jr., “Gravitational Wave Generator,” United States Patent Number 6,417,597 B1, 2002.

Baker, R.M.L., Jr., Ed., Proc. Gravitational-Wave Conference, MITRE Corp., McLean, VA (2003a).

Baker, R.M.L., Jr., "Generation of High-Frequency Gravitational Waves (HFGWs) by Means of an Array of Micro- and NanoDevices," in Proc. Gravitational-Wave Conference, MITRE Corp., McLean, VA, HFGW-03-117 (2003b).

Baker, R.M.L., Jr., "Precursor Experiments Regarding the Generation of High-Frequency Gravitational Waves (HFGWs) by Means of Using an Array of Micro- and Nano-Devices," in Proceedings of the Space Technology and Applications International Forum (STAIF-04), edited by M.S. El-Genk, AIP Conference Proceedings 699, Melville, NY, 2004, pp. 11061113.

Baker, R.M.L., Jr., Davis, E.W. and Woods, R.C., "Gravitational Wave (GW) Radiation Pattern at the Focus of a HighFrequency GW (HFGW) Generator and Aerospace Applications," in these proceedings of Space Technology and Applications International Forum (STAIF-05), edited by M.S. El-Genk, American Institute of Physics, Melville, NY, 2005.

Chincarini, A. and Gemme, G., "Micro-wave Based High-Frequency Gravitational Wave Detector," in Proc. Gravitational-Wave Conference, MITRE Corp., McLean, VA, HFGW-03-103 (2003).

Chincarini A., private communication (2003).

Cruise, A.M., "An Electromagnetic Detector for Very-High-Frequency Gravitational Waves," Classical and Quantum Gravity 17, 2525-2530 (2000).

Einstein, A., “Zür allgemeinen Relativitätstheorie,” Sitzungsberichte, Preussische Akademie der Wisserschaften, 778-786 (1915).

Einstein, A., "Näherungsweise Integration der Feldgleichungen der Gravitation," Sitzungsberichte, Preussische Akademie der Wisserschaften, 688-696 (1916).

Einstein, A. and Rosen, N., "On Gravitational Waves," Journal of the Franklin Institute 223, 43-54 (1937).

Gordon, F.E., private communication, Navy SPAWAR Systems Center, San Diego, Ca. (2004).

Hawking, S.W. and Israel, W. General Relativity: An Einstein Centenary Survey, CUP, Cambridge, 1979, Ch. 3 , p. 98.

Kowitt, M., "Gravitomagnetism and Magnetic Permeability in Superconductors," Phys. Rev. B 49, 704-708 (1994).

Lakin, K.M., Belsick, J., McDonald, J.F. and McCarron, K.T., "High Performance Stacked Crystal Filters for GPS and Wide Bandwidth Applications," in Proc. IEEE Ultrasonics Symposium, IEEE, Piscataway, NY, 2001, pp. 833-838.

Landau L.D. and Lifshitz E.M., The Classical Theory of Fields, 4th Revised English Edition, Pergamon, Oxford, 1975, pp. 348, $349,355-357$.

Li, N. and Torr, D.G., "Gravitational Effects on the Magnetic Attenuation of Superconductors," Phys. Rev. B 46, 5489-5495 (1992).

Li, N., private communication (2002).

Prince, T.A., private communication (2002).

Ruby, R., Bradley, P., Larson, J.D., and Oshmyansky, Y., "PCS 1900MHz Duplexer Using Thin Film Bulk Acoustic Resonators (FBARs)," Elec. Lett. 35, 794-795 (1999).

Ruby, R. and Merchant, P., "Micromachined Thin Film Bulk Acoustic Resonators," in Proc. 48th IEEE Int. Frequency Control Symposium, IEEE, Piscataway, NY, 1994, 135-138.

Saleh, B.E.A. and Teich, M.C., Fundamentals of Photonics, Wiley, New York, NY, 1991, p. 95.

Taylor, J.H., "Binary Pulsars and Relativistic Gravity," Rev. Modern Phys. 66, 711-719 (1994).

Weber, J., "Gravitational Waves," in Gravitation and Relativity, Benjamin, New York, NY, 1964, Chapter 5, pp. 90-105. 